\title{
Methods for Assessing the Seismic Resistance of Subterranean Hydro Structures Under the Influence of Seismic Waves
}

\author{
Safarov Ismail Ibrahimovich ${ }^{1}$, Boltayev Zafar Ixtiyorovich ${ }^{2}$ \\ ${ }^{1}$ Department of «Higher Mathematics», Tashkent Chemistry - Technological Institute, Tashkent, Republic of Uzbekistan \\ ${ }^{2}$ Department of «Higher Mathematics», Bukhara Technological Institute of Engineering, Bukhara, Republic of Uzbekistan
}

Email address:

safarov54@mail.ru (S. I. Ibrahimovich)

To cite this article:

Safarov Ismail Ibrahimovich, Boltayev Zafar Ixtiyorovich. Methods for Assessing the Seismic Resistance of Subterranean Hydro Structures Under the Influence of Seismic Waves. American Journal of Physics and Applications. Vol. 6, No. 2, 2018, pp. 51-62.

doi: 10.11648/j.ajpa.20180602.14

Received: December 20, 2017; Accepted: February 5, 2018; Published: February 23, 2018

\begin{abstract}
The paper considers the seismic resistance of subterranean hydro constructions of various shapes under the influence of seismic waves. The review is dedicated to the abovementioned issues. Recommendations on increasing seismic resistance of underground hydraulic structures under the action of seismic waves are suggested.
\end{abstract}

Keywords: Wave, Hydro Construction, Seismic Resistance, Liquid, Wavelength, Frequency

\section{Introduction}

Modern automobile, railway and hydro technical tunnels buried in the ground trunk pipelines in accordance with the requirements of reliability and durability for them are among the most important objects of underground construction. No less important are urban underground structures. In essence, modern cities grow not only upward, but also downwards, using underground space, which facilitates the separation of transport and pedestrian flows, transit and local, high-speed and conventional transport. Extremely widespread development of the construction of underground main pipelines, providing transportation of virtually the entire volume of natural gas produced in the country. Predictive assessments of the behavior of tunnel structures and underground pipelines under dynamic impacts in real conditions of construction and operation, which should be carried out at the stage of their design, are determined by the stress-strain state of the structure in interaction with the surrounding rock or the earth stratum. Among the dynamic impacts, a special place is occupied by the effects of earthquakes that are affecting the construction area, as well as repeated industrial explosions during the tunneling workings.

The existing methods for calculating underground structures, analog accelerograms, can be successfully divided into two main groups, based on different schematization, both the structures themselves and their interaction with the environment. One of these directions is the seism dynamic theory of complex systems of underground structures, developed in $[32,38,39,40]$, develops in relation to pipelines and tunnels of subways.

In these works it is assumed that the extended branching structure has rigid or compliant knots in the branching areas and is schematized by a set of rigid beam structures interacting with the ground with six degrees of freedom. The sections of pipelines or tunnels interlocking with each other, interacting with the ground and nodal structures, are considered as beams working on tension-compression, bending and torsion. When considering the interaction with the soil, a number of rheological models of the soil are considered. In this connection, a great deal of experimental work was carried out to study the interaction of various pipes and lining with soil. Analysis of the solutions [4] obtained for transverse vibrations of elements of single-track tunnels from circular solid sectional lining interacting with an ideally elastic soil showed that ground conditions significantly affect the values of the first few frequencies of natural oscillations.

When calculating the seismic dynamic theory, the 
maximum bending moment of the lining is greater than that obtained when calculating according to the static theory. Numerical calculations performed for specific examples of metro linings have made a number of constructive conclusions. For example, in the case of a rigid jamming of the base (massive base), significant stresses occur near the jamming, a solid side filling of the walls and a soft backfilling of the crossbars, as compared with the case of solid backfilling, leads to an increase in natural frequencies. Seism dynamic theory allows us to consider also longitudinal oscillations of tunnels [1, 2, 33, 34] and pipelines [32, 38]. This direction is also developed in $[35,36,37]$. Taking into account the interaction of the structure with the soil, concluded that this is measured by a nonlinear law.

Another way to determine the seismic state of structures of underground structures when calculating them for analog accelerograms is associated with the use of wave dynamics methods. Tunnels and underground pipelines are extensive underground structures; such are the mine workings of mines and mines such as drifts, overhangs and diagonal workings.

\section{Estimation of Seismic Stress of Underground Structures by Wave Dynamics Methods}

In the case of a sufficiently long cavity and an impact directed perpendicular to the longitudinal axis, the surrounding medium cavity and the lining are in a plane deformation state, and the problems of determining the stress state of the array and lining are reduced to the plane problem of the dynamic theory of elasticity. In view of the fact that the length of seismic waves, as a rule, exceeds the characteristic dimensions of the cross sections of the excavations (for example, the diameter D), solutions of diffraction problems for long-wavelength effects are of particular interest. when $D / \lambda<1$.

In $[5,13,16,17,18]$, problems of stress concentration in an unbounded linearly-elastic plane near a circular cavity of diameter $\mathrm{D}$ in the propagation of longitudinal harmonic waves with a length $\lambda$. The maximum coefficients of dynamic stress concentrations $K_{\sigma}$ the ratio of the maximum stresses on the contour of the hole to the amplitude of the incident plane wave) is assumed for large wavelengths $\left(\frac{D}{\lambda}=0,04 \div 06\right)$ those. the maximum coefficients of dynamic concentrations turned out to be $5-10 \%$ higher than with the corresponding biaxial static loaded $\left(\lambda_{\infty}\right)$. If $\frac{D}{\lambda}>0.16$, then the dynamic stress concentrations are significantly lower than the static stresses. Solutions of the diffraction problem for a plane harmonic transverse wave were obtained in $[25,26$, 27]. In the field of "dynamic ejection" the voltage is $10-15 \%$ higher than statically.

One of the problems is devoted to the propagation of harmonic shear waves in a two-dimensional elastic body with a circular aperture (reinforced). In this formulation, the imposition of suitable waves and the shear and stretchingcompression waves reflected from the aperture is studied, which leads to stress concentration. The solution of the diffraction problem for a plane harmonic shear wave was obtained in [28], which has the following form $\left(\sigma_{\theta \theta}^{*}=\sigma_{\theta \theta}{ }^{\prime}\right.$ $\sigma_{0} ; \sigma_{\theta}=\mu \beta^{2} \psi_{0} ; \psi_{0}$ - amplitude of the incident waves, $\mu$ Lame coefficient)

$$
\sigma_{\theta \theta}^{*}=\frac{8}{\pi}\left(1-\frac{1}{n^{2}}\right) \sum_{n=1}^{\infty} i^{n} \frac{n\left(n^{2}-1-\frac{\beta^{2} \alpha^{2}}{2}\right) H_{n}(\alpha a)}{\Delta_{n}} \sin n \theta e^{-i \omega t}
$$

where

$$
\begin{aligned}
& \Delta_{n}=\alpha a H_{n-1}(\alpha a)\left[\left(n^{2}-1\right) \beta a H_{n-1}(\beta a)-\left(n^{3}-n+\frac{1}{2} \beta^{2} \alpha^{2}\right) H_{n}(\beta a)\right]+ \\
& +H_{n}(\alpha a)\left[-\left(n^{3}-n+\frac{1}{2} \beta^{2} \alpha^{2}\right) \beta a H_{n-1}(\beta a)+\left(n^{2}-n-\frac{1}{4} \beta^{2} \alpha^{2}\right) \beta^{2} \alpha^{2} H_{n}(\beta a)\right]
\end{aligned}
$$

$\mathrm{H}_{\mathrm{n}}(\beta a)-$ Hankel function; $\alpha=\omega / \mathrm{C}_{\mathrm{p}} ; \beta=\omega / \mathrm{C}_{\mathrm{S}} ; \mathrm{C}_{\mathrm{p}}$ and $\mathrm{C}_{\mathrm{S}}$ - respectively, the propagation velocity of longitudinal and transverse waves; $\omega$ - circular frequency, $\pi=3,14$. Calculations by the methods of the theory of elasticity give, in the absence of lining around the hole and the wavelength, substantially larger than the hole diameter, the following approximate expression for the perimeter stresses

$$
\sigma_{\theta \theta}=\frac{2 G v_{0}}{c_{S}}\left(1-c_{S}^{2} / c_{p}^{2}\right) \sin 2 \theta \sin \omega t
$$

where $G$ - shear modulus for soil, $v_{0}$ - amplitude of the velocity of the incident seismic waves. In view of the fact that long seismic waves tend to exceed the characteristic dimensions of transverse cut-off workings (for example, diameter D), the solution of diffraction problems for longwavelength effects is of particular interest. when $D / \lambda<1$.

The maximum coefficients of dynamic stress concentrations $K_{\sigma}$ (the ratio of the maximum stresses on the contour to the amplitude of the incident plane wave) in Figure 1 , depending on the values of the parameters $D / \lambda$ and the Poisson's ratio $v$ of the plane material. 


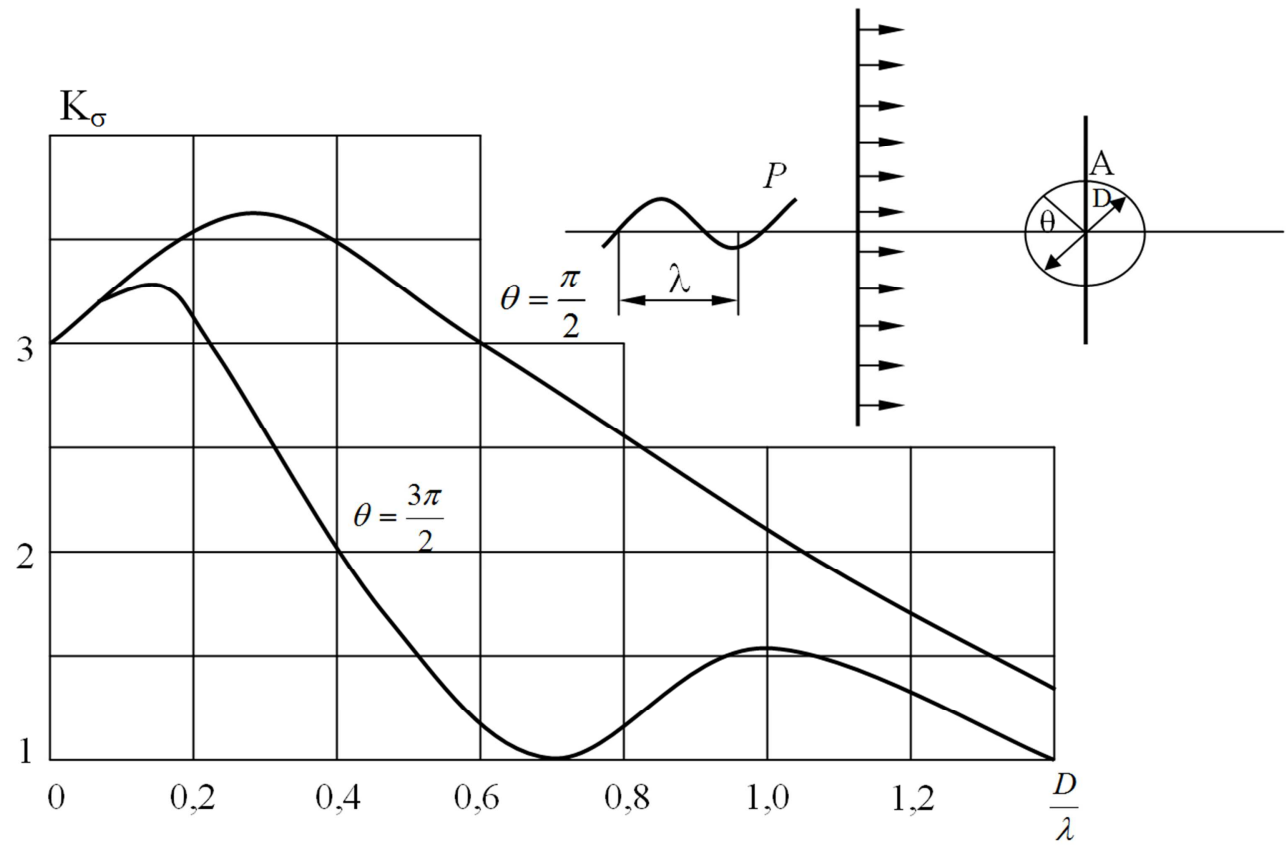

Figure 1. Dependence of the voltage concentration factor on the wavelength.

The depth of the underground hydro technical structures, as a rule, is small in comparison with the seismic wave lengths. In this case, the solutions of dynamic problems should be influenced by the free surface. Therefore, the results of studying the diffraction of stress waves in semi bounded arrays with cylindrical cavities are of interest. In [7, $8,10]$, problems of diffraction of stationary transverse waves on a circular cavity with a diameter of $\mathrm{D}$ were solved. The results obtained indicate that the distribution of the stress concentration in the hole depends very strongly on the length of the incident waves, the direction of its propagation, and the depth of deposition. To calculate the tunnel with hard lining in soft ground in works $[3,12,15]$ accepted models of inclusion of elastic material in elastic environment. The problem of the diffraction of a plane harmonic $\mathrm{P}$ wave on the circular solid inclusion of the diameter D-is considered in Ref. 19 for cases of rigid coupling between the inclusion and the cavity (hard sealing) and some slippage of the elastic washer. With dynamic emissions reaching 200-300\%, the stresses of long-wave effects exceed the solutions of the static problem $[19,20]$ several times.

Increasing the stiffness of the inclusion leads to an increase in the maximum tangential stresses in the array at the boundary with the switch. To determine the seismic state of tunnels, solutions of dynamic problems on stress concentrations on circular cylindrical shells located in elastic massifs are of the greatest interest. The work [3, 12, 15] is devoted to the interaction of harmonic P-waves with the tunnel, taking into account the presence of water in the tunnel. For the case of lining in the form of a thin bonding ring, formulas are obtained that allow determining the stresses and displacements in the lining, and also the displacement in the array on the contour of the lining. The propagation of elastic waves in multiply connected regions, in the case of a plane problem, significantly affects the structure's VAT. The solution of these problems requires, first of all, the involvement of wave dynamics methods, since interference effects, which make the main contribution to the dynamic stress state of a multiply connected region, can not be taken into account in other ways. The analysis of problems on the dynamic stressed state of multiplyconnected domains in the propagation of elastic waves is devoted to the papers $[19,20]$. When a plane harmonic longitudinal wave $P$ falls by an infinite length of circular holes with a diameter D located in a linearly elastic plane parallel to the wave front, the stress distribution between the holes is investigated, depending on the values of the parameter D and the distance between the centers of the holes $\delta$.

The nature of the stress state of the zone between the holes when fixing $\delta$ is determined by the parameter value $\mathrm{D} / \lambda$ similar to diffraction in a single hole (dynamic ejection, etc.)

In the long-wave range $(D / \lambda \leq 0,2)$ for a fixed $\delta$ or with decreasing $\delta$ (for a fixed $\mathrm{D} / \lambda$ ) there is alignment of the jumpers, as a result of which the total stresses in the region between the holes can significantly exceed the stresses on the contours of the holes. The values of the voltages on the free contours of the holes (for all values $\delta$ ) have a maximum at the same values $\mathrm{D} / \lambda$. These maxima exceed the corresponding static stresses (under biaxial stretching of the plane by conditions $\left(\mathrm{P}_{\mathrm{x}}\right.$ and $\left.\mathrm{P}_{\mathrm{y}}=0.3 \mathrm{P}_{\mathrm{x}}\right)$ by about $18-20 \%$, creating another "dynamic outlier", determined by the values $\delta$.

Similar conclusions can be drawn from the solution of the same problem for a transverse wave. In pressure tunnels and water canals, the seismic pressure of water is directed to the lining [20, 44]. In this connection, the solution of the problem of a plane with two identical round holes (diameter 
of the holes $\mathrm{D}$, the distance between their centers $\delta$ ), to the contours of which the harmonic pressure is applied (from the inside). To this problem is reduced (in a stationary setting) the investigation of the seismic stress state of lining and inter-chamber ends of two threads parallel to the pierced water conduits. Defined by the parameters of the task are D/ $\lambda$ and $\delta / \lambda$. When $D / \lambda=0,06 \div 0,1$ The contour stresses are maximal, for all $\delta$ exceeding static values by

$18-20 \%$ [17]. In [29], static and dynamic calculations of underground parallel pipes interacting with the ground environment are considered. Static calculations are performed numerically, using the finite element method. In work [45, 47] stress-strain states of cylindrical tubes with a liquid are considered, under the influence of seismic waves. The most interesting is the reaction of underground structures to non-stationary (impulse) impacts.

As is known, [43, 50], according to the reaction of the structure to any impulse action, it is possible to determine the reaction to any other nonstationary action. Thus, it is possible to obtain the necessary information for the calculation of underground structures directly to a given seismic action of an arbitrary kind, for example, a real accelerogram. Considering the non-stationary effects, we take into account the phase of the wave entry dangerous for the designs. In [39, $40,41]$, a comparison of the concentration coefficients $K_{o}$, obtained from the results of studies of the interaction of a reinforced cavity with a harmonic longitudinal wave and obtained for the same problem, but for a stepwise pressure wave. The relatively thin $(h / D=0,025)$ and massive $(h / D=0,09)$ lenses from materials with greater than the environment, stiffness n. Concentration coefficients (the level of "dynamic ejection") in the linearly elastic formulation of the nature of the loaded (in the long-wave region) are almost independent. In [42], the influence of the parameters $\mathrm{n}$ and $h / D$ to the level $K_{O}$ in the diffraction of $\mathrm{P}$ waves on cylindrical shells in linearly elastic media. In [43] the dependence $K_{o}=f(D / h, n)$ It is established that the general nature of the dependences $K_{o}=$ $(D / \lambda)$ However, the numerical values $K_{a}$ are predominantly determined by the value of the parameter $n$. Concentration of stresses on the internal, free contour of relatively rigid $(n>1)$ lining can exceed by an order of magnitude or more $K_{a}$ for compliant $(\mathrm{n}<1)$ lining. At the present time, theoretical methods for solving problems on the diffraction of no stationary stress waves on cavities of various shapes have been insufficiently developed [44, 45] and are limited by the capabilities of computer technology. In this connection, experimental methods for investigating diffraction problems, as well as determining the seismic stress of specific underground structures, have become very popular.

Most of the work was done in a linear-elastic formulation using the dynamic photo-elastic method [46]. In most cases, the experimental difficulties did not allow us to investigate in detail the diffraction problems in the long-wavelength range, which is most interesting for seismic stability problems. Only in a few works, in the field $(D / \lambda \leq 0,2)$ the experimental values of the maximum stress concentrations, recalculated for a stepwise action, fit well into the theoretical dependence obtained for stationary and nonstationary effects. In [46], it was possible to obtain the values of the parameter $\frac{D}{\lambda}\left(0,9 \leq \frac{D}{\lambda} \leq 1,5\right)$ on the basis of the experimental results "dynamic emission". Experiments were carried out for a transverse wave in the short-wavelength range. The experimental points fit well on the curve, [40, 47, 48], which once again confirms the insignificance of the differences in $K_{o}$ for stationary and no stationary effects in a linearly elastic infinite cavity. The effect of the shape of the hole on the value was investigated [49] for square and vaulted cavities with rounded straight angles under the action of $\mathrm{P}$ and $\mathrm{S}$ waves. It turned out that in the vault the stress distribution is close to the distribution for the circular hole. Thus, in the investigated range of short-wave voltage near the vaulted hole. Stresses under the influence of longitudinal and transverse waves for a circular and square hole can be determined on the basis of solving diffraction problems. The effect of the reinforced contour of the hole on the flat models was investigated in $[44,50]$. It is shown that in the case of reinforced holes, the nature of the dependence $K_{\delta}$ of the parameter $D / h$. Remains the same as for unsupported cavities. When diffraction of short $(D / h=0,64)$ waves on circular holes on the "illuminated" side there is an overstressed zone as a result of the imposition of incident and reflected waves. The maximum stress value is greater than 2 times than the amplitude value of the longitudinal component of the incident pulse. The reinforcement of the contour of the hole with lining, the acoustic rigidity of which is much larger than that of the environment $(n=0.16)$, significantly changes the stress distribution in the "illuminated" zone. The case of the action of longer $\mathrm{P}$ waves $(D / \lambda=0,25 \div 0,6)$ on a circular hole with a rigid reinforcing lining $(n=0.08$ and $n=$ $0.16)$ was considered in [50]. The obtained results indicate that the concentration of stresses on the free contour of the lining is substantially (almost twice) higher than the value $K_{\delta}$ for an unsupported cavity, but the presence of a lining reduces the concentration of stresses in the massif by 20 $40 \%$. It is also found that increasing the rigidity of the lining leads to an increase in stress concentrations. That most soils are known to exhibit dynamic inelastic effects, such as many cohesive soils, behave as an essentially visco elastic medium $[42,43]$. Elastic idealization in solving diffraction problems for such media may not be accurate enough. In [42] numerical results are presented for the case of diffraction of stepwise waves of tension and shear on a concrete cylinder placed in sandstone, which is characterized by the properties of a standard linear body. When calculating the following: the relative thickness of the lining $\frac{h}{D}=0,05$, the ratio of sandstone and concrete densities is 0.987 . The dynamic effect of explosions on the development depends on their mutual arrangement. If the explosions are produced close to the workings, then the waves are reflected from their surface and the stresses of the opposite sign appear in the mass surrounding the workings, i.e. the main energy of the 
explosion is concentrated in the compression phase [41, 46]. If the source of the explosive effect from the cavity is sufficiently distant $\left(\mathrm{r}>5 \Gamma_{0}\right)$ The solutions of the diffraction problems for plane waves considered above are valid.

The study of the natural vibrations of bodies associated with an infinite environment is of great importance in the national economy. The authors of $[6,11]$ carried out an analysis of the frequencies and modes of oscillations of an infinite cylindrical shell contacting with an inertial medium.

One of the main difficulties arising in the problems of geophysics, seismic exploration, and problems of calculating oscillations in an infinite medium is integration into infinity. For the transition from an infinite region to a finite one, the introduction of external friction is used. For example, in [22] it was shown that for longitudinal oscillations of a composite semi-infinite elastic rod, the spectral problem is equivalent to the problem of the natural vibrations of a finite rod with external damping of damper type. This method - the introduction of external friction - has also been used to determine the periods of natural oscillations of the chimney [7], consideration of forced oscillations of the axial symmetric viscoelastic cylindrical shell [39, 49, 50], the intrinsic and forced oscillations of the model of the construction-ground system [7, 8], spatial forms of oscillations of the axis of symmetric structures [31].

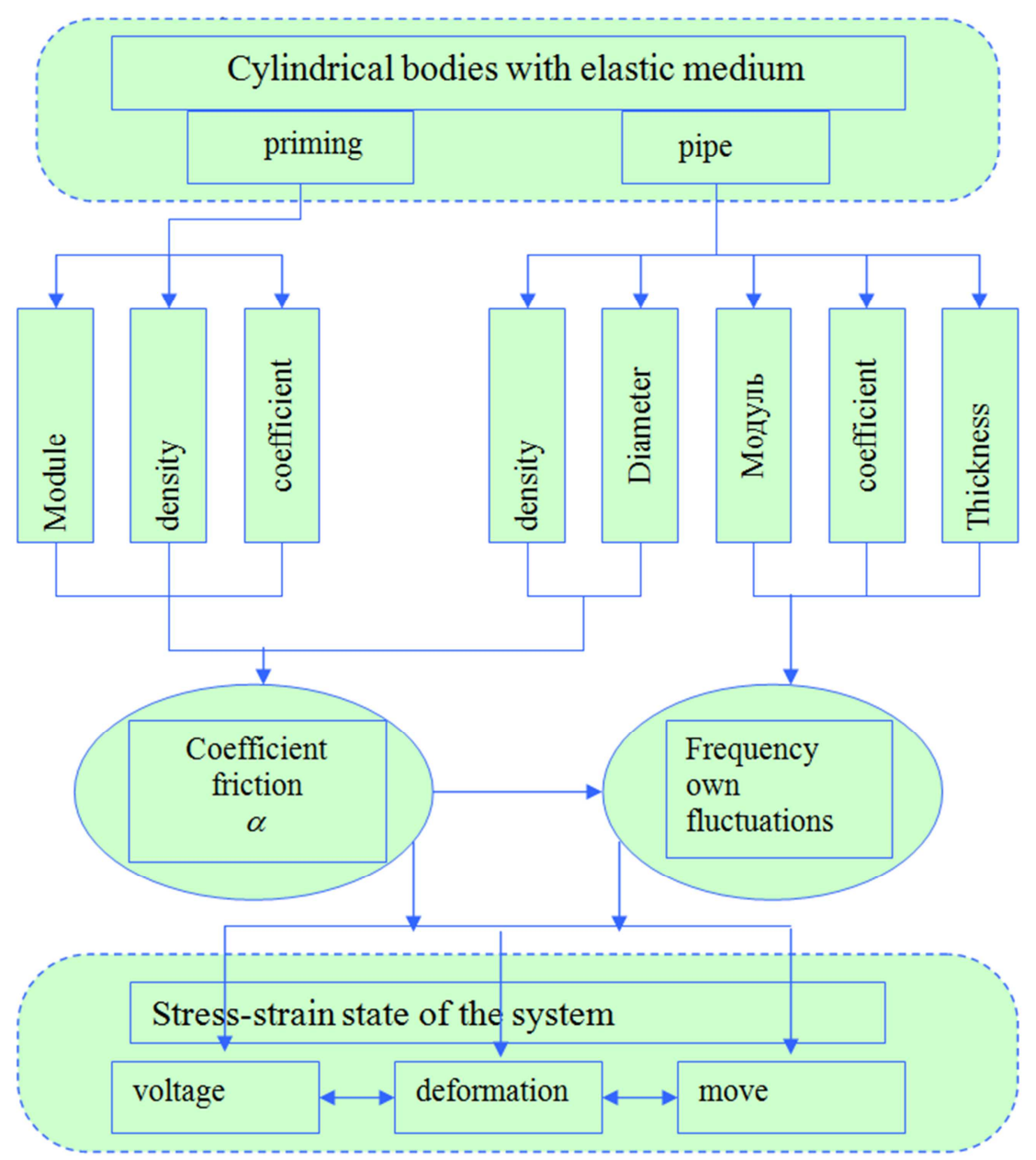

Figure 2. Investigation of the parameter of natural oscillations of cylindrical bodies with an elastic medium.

In $[29,30]$, the natural oscillations of a cylindrical layer in an elastic, infinite medium are investigated. The problem is solved numerically and analytically in a flat formulation. The solution of the problem of natural oscillations of rectangular bodies in an elastic medium is considered in [31]. The problem is solved in a flat formulation by the method of separated variables. In this case, the natural frequencies are complex. The imaginary parts of the eigen frequencies denote the attenuation coefficient. The real and imaginary parts of the complex natural frequency have not been studied sufficiently.

\section{The Intrinsic Oscillations of Pipelines in an Elastic Medium with Specific Parameters}

When the forces act, the pipeline leaves the equilibrium position, i.e. Deformation occurs (stretching, shear).

The pipeline is moved in the longitudinal direction by a distance depending on the interaction of the soil surrounding the pipe with the surface of the pipe.

The interaction of soil with the surface of the pipe is 
determined through the interaction $\alpha$ (Figure 2).

In this section we present the results of calculating the friction coefficient of pipelines with the environment and the natural oscillation frequency of the pipelines used at the design stage.

Suppose that an infinitely long rectilinear underground pipeline is pinched by the surrounding soil, which has elastic properties. From the source of the earthquake, seismic waves propagate in the ground. The pipeline, connected with the ground, is involved in the oscillation process. However, the soil transfers forces to the pipeline with some slippage, i.e. It is impossible to assume that with significant soil displacements, there will not be a breakdown along the pipeground boundary. Thus, the relative displacements in the ground environment $\varepsilon_{z p}$ will be greater than the relative offset $\varepsilon_{m p}$ conduit, i.e. $\varepsilon_{m p}=\alpha \varepsilon_{z p}$, where $\alpha$ - coefficient of interaction (Figure 2).
With the increase in the diameter of the pipeline, the degree of its interaction (pinching) in the same ground is reduced, so with increasing pipeline diameter, longitudinal stresses decrease, and bending stresses increase. The interaction of the pipeline will also decrease with a decrease in the rigidity of the soil, which will affect the stresses in the pipeline accordingly. The length of the pipeline affects the stresses for sections whose length is commensurate with the length of the seismic wave. The coefficient of friction is defined as follows:

$$
\alpha=\frac{9 \rho^{*}\left(2 \chi^{3}+1\right)}{\tau_{0}^{*}\left(2 \chi^{2}+1\right)^{2}}
$$

where

Table 1. Values $\alpha$ for certain soil and pipe parameters.

\begin{tabular}{|c|c|c|c|c|c|}
\hline Relationships & $\rho\left(10^{3} \mathrm{Gz} / \mathrm{m}^{3}\right)$ & $E\left(10^{11} \mathrm{Gz} / \mathrm{m}^{2}\right)$ & d $(\mathbf{m})$ & $\mathbf{v}$ & $\alpha$ \\
\hline Sandstone & 25 & 0,17 & & 0,3 & \multirow{2}{*}{0,283} \\
\hline Steel & 78 & & 1,2 & & \\
\hline Clay & 16 & 0,3 & & 0,41 & \multirow{2}{*}{0,107} \\
\hline Steel & 78 & & 1,2 & & \\
\hline Concrete & 18 & 0,2 & & 0,17 & \multirow{2}{*}{0,269} \\
\hline Steel & 78 & & 1,2 & & \\
\hline Rubber & 9 & 0,00008 & & 0,47 & \multirow{2}{*}{0,023} \\
\hline Steel & 78 & & 1,2 & & \\
\hline Sandstone & 25 & 0,17 & & 0,3 & \multirow{2}{*}{0,484} \\
\hline Steel & 78 & & 0,7 & & \\
\hline Clay & 16 & 0,3 & & 0,41 & \multirow{2}{*}{0,183} \\
\hline Steel & 78 & & 0,7 & & \\
\hline Concrete & 18 & 0,2 & & 0,17 & \multirow{2}{*}{0,461} \\
\hline Steel & 78 & & 0,7 & & \\
\hline Rubber & 9 & 0,00008 & & 0,47 & \multirow{2}{*}{0,039} \\
\hline Steel & 78 & & 0,7 & & \\
\hline Sandstone & 25 & 0,17 & & 0,3 & \multirow{2}{*}{0,316} \\
\hline Cast iron & 70 & & 1,2 & & \\
\hline Concrete & 18 & 0,2 & & 0,17 & \multirow{2}{*}{0,302} \\
\hline Cast iron & 70 & & 1,2 & & \\
\hline Sandstone & 25 & 0,17 & & 0,3 & \multirow{2}{*}{0,886} \\
\hline Asbestos & 25 & & 1,2 & & \\
\hline Concrete & 18 & 0,2 & & 0,17 & \multirow{2}{*}{0,843} \\
\hline Asbestos & 25 & & 1,2 & & \\
\hline Sandstone & 25 & 0,17 & & 0,3 & \multirow{2}{*}{1,261} \\
\hline Cast iron & 70 & & 0,3 & & \\
\hline Concrete & 18 & 0,2 & & 0,17 & \multirow{2}{*}{1,204} \\
\hline Cast iron & 70 & & 0,3 & & \\
\hline Sandstone & 25 & 0,17 & & 0,3 & \multirow{2}{*}{3,531} \\
\hline Asbestos & 25 & & 0,3 & & \\
\hline Concrete & 18 & 0,2 & & 0,17 & \multirow{2}{*}{3,366} \\
\hline Asbestos & 25 & & 0,3 & & \\
\hline
\end{tabular}

$$
\begin{gathered}
\rho^{*}=\frac{\rho_{z p}}{\rho_{c m}} ; \chi=\frac{C_{p}}{C_{s}} ; \\
C_{p}=\sqrt{E_{z p} g\left(1-v_{z p}\right) /\left[\rho_{z p}\left(1+v_{z p}\right)\left(1-2 v_{z p}\right)\right] ;} \\
C_{s}=\sqrt{E_{z p} g /\left[2 \rho_{z p}\left(1+v_{z p}\right)\right]} ; \tau_{0}^{*}=d \chi
\end{gathered}
$$

$\rho_{\Gamma P}$ - soil density; $\rho_{C T}$ - steel density; $C_{P}$-velocity of longitudinal waves; $C_{S}$ - transverse wave velocity; $E_{\Gamma P}$ - modulus of 
soil elasticity; $v_{\Gamma Р}$ - Poisson's coefficient of soil; $\mathrm{g}$ - acceleration of gravity; $\mathrm{d}$ is the diameter of the tube.

Some values $\alpha$ obtained with certain soil parameters and pipes are given in Table 1.

Table 2. Frequency results for natural oscillations。

\begin{tabular}{|c|c|c|c|c|c|c|}
\hline Environment & & & Pipe $(d=0.7)$ & & & $\omega_{1} \mathrm{rad} / \mathrm{s}$ \\
\hline Sandstone & & & Сталь & & & \multirow{3}{*}{4,8587} \\
\hline $\mathrm{E}\left(10^{11} \mathrm{Gz} / \mathrm{m}^{2}\right)$ & $v$ & $\rho \quad\left(10^{3} \mathrm{Gz} / \mathrm{m}^{3}\right)$ & $\mathrm{E}\left(10^{11} \mathrm{Gz} / \mathrm{m}^{2}\right)$ & $v$ & $\rho\left(10^{3} \mathrm{Gz} / \mathrm{m}^{3}\right)$ & \\
\hline 0,17 & 0,3 & 2,5 & 2,1 & 0,25 & 7,8 & \\
\hline Clay & & & Steel & & & \multirow{2}{*}{3,6742} \\
\hline 0,3 & 0,41 & 1,6 & 2,1 & 0,25 & 7,8 & \\
\hline concrete & & & Steel & & & \multirow{2}{*}{4,9582} \\
\hline 0,2 & 0,17 & 1,8 & 2,1 & 0,25 & 7,8 & \\
\hline Rubber & & & Steel & & & \multirow{2}{*}{0,7192} \\
\hline 0,00008 & 0,47 & 0,9 & 2,1 & 0,25 & 7,8 & \\
\hline Sandstone & & & Cast iron & & & \multirow{2}{*}{5,1319} \\
\hline 0,17 & 0,3 & 2,5 & 1,3 & 0,24 & 7,0 & \\
\hline concrete & & & Cast iron & & & \multirow{2}{*}{5,2576} \\
\hline 0,2 & 0,17 & 1,8 & 1,3 & 0,24 & 7,0 & \\
\hline Sandstone & & & Asbestos & & & \multirow{2}{*}{8,5890} \\
\hline 0,17 & 0,3 & 2,5 & 0,3 & 0,17 & 2,5 & \\
\hline concrete & & & Asbestos & & & \multirow{2}{*}{8,7831} \\
\hline 0,2 & 0,17 & 1,8 & 0,3 & 0,17 & 2,5 & \\
\hline
\end{tabular}

\section{Determination of the Dynamic Stress- Strain State of a Cylindrical Tube Under the Action of a Seismic Type}

After determining the coefficient of friction $\alpha$, determine the frequencies of the natural oscillations of the tube.

$$
\omega^{2}=\frac{9 \rho^{*}}{\tau_{1}\left(2 \chi^{2}+1\right)}
$$

where

$$
\tau_{1}=\sqrt{\frac{d}{C_{p}}}
$$

Some of the results obtained for the frequencies of the natural oscillations of the pipes for various soil parameters are given in Table 2 .

From the frequencies found, it is possible to determine the displacements, as well as the stresses in the pipelines arising from their own oscillations (Figure 1). The results obtained can be used to design the linear part of new and reconstructed pipelines and branches from them.

The addition theorems for cylindrical wave functions are derived in [9]. Let there be two different polar coordinate systems $\left(\mathrm{r}_{\mathrm{g}}, \theta_{\mathrm{g}}\right)$ and $\left(\mathrm{r}_{\mathrm{k}}, \theta_{\mathrm{k}}\right)$ ( 3 and 4$)$, in which the polar axes are equally directed. Pole coordinate $\theta_{\mathrm{k}}$ in q system will $\mathrm{R}_{\mathrm{kq}}$, $\theta_{\mathrm{kq}}$, so that equality

$$
Z_{g}=R_{k g} e^{i \theta_{k g}}+Z_{k}
$$

Then the addition theorem has the form:

$$
\begin{aligned}
& b_{n}\left(\alpha r_{q}\right) e^{i n \theta_{q}}=\sum_{p=-\infty}^{\infty} b_{n-p}\left(\alpha R_{k q}\right) e^{i(n-p) \theta_{k q}} T p\left(\alpha r_{k}\right) \exp \left(i p \theta_{k}\right), \\
& r_{k}<R_{k q} ; \\
& b_{n}\left(a r_{q}\right) e^{i n \theta_{q}}=\sum_{p=-\infty}^{\infty} J_{n-p}\left(\alpha R_{k q}\right) e^{i(n-p) \theta_{k q}} b_{p}\left(\alpha r_{k}\right) \exp \left(i p \theta_{k}\right), \\
& r_{k}<R_{k q} ;
\end{aligned}
$$

Formulas (1) make it possible to transform the solution of the wave equation from one coordinate system to another.

Consider the calculation of an extended underground multi-thread pipeline for seismic action within the framework of the plane problem of the dynamic theory of elasticity. In this case, we investigate the case of stationary diffraction of plane waves on a series of periodically located cavities, supported by rings with an ideal compressible fluid inside.

The solution of the problem is realized by the method of potentials. The form of the incident potential will not change either. The potentials of the waves reflected from the tubes after the application of the addition theorem and, taking into account the periodicity of the problem, will have the form:

$$
\begin{gathered}
\left.\varphi_{1}^{(r)}=e^{-i w t} \sum_{n=0}^{\infty}\left[A_{n} H_{n}^{(1)}\left(\alpha_{1} r\right)+S_{n} J_{n}\left(\alpha_{1} r\right)\right]\right]^{i n(\theta-\gamma)}, \\
\left.\psi_{1}^{(r)}=e^{-i w t} \sum_{n=0}^{\infty}\left[B_{n} H_{n}^{(1)}\left(\beta_{1} r\right)+\sigma_{n} J_{n}\left(\beta_{1} r\right)\right]\right]^{i n(\theta-\gamma)}, \\
S_{n}=\sum_{p=0}^{\infty} \sum_{m=1}^{\infty} A_{p} E_{p}\left[e^{i m \xi} H_{n-p}^{(1)}\left(\alpha_{1} m \delta\right)+e^{-i m \xi} H_{n-p}^{(1)}\left(\alpha_{1} m \delta\right)\right] \\
Q_{n}=\sum_{p=0}^{\infty} \sum_{m=1}^{\infty} B_{p} E_{p}\left[e^{i m \xi} H_{n-p}^{(1)}\left(\beta_{1} m \delta\right)+e^{-i m \xi} H_{n-p}^{(1)}\left(\beta_{1} m \delta\right)\right]
\end{gathered}
$$

where: $\xi=\mathrm{k} \delta \cos \gamma, \delta$ - distance between pipe centers. 
The potentials of refracted waves in the pipes will be written in the form

$$
\begin{aligned}
& \varphi_{2}=e^{i(m \xi-w \xi)} \sum_{n=0}^{\infty} E_{n}\left[C_{n} H_{n}^{(1)}\left(\alpha_{1} r\right)+D_{n} H_{n}^{(2)}\left(\alpha_{2} r\right)\right] e^{i n(\theta-\gamma)}, \\
& \psi_{2}=e^{i(m \xi-w \xi)} \sum_{n=0}^{\infty} E_{n}\left[E_{n} H_{n}^{(1)}\left(\beta_{1} r\right)+F_{n} H_{n}^{(2)}\left(\beta_{2} r\right)\right] e^{i n(\theta-\gamma)}
\end{aligned}
$$

and the velocity potential in the ideal form of a compressible fluid

$$
\varphi_{3}=e^{i(m \xi-w \xi)} \sum_{n=0}^{\infty} E_{n} G_{n} J_{n}\left(\alpha_{3} r\right) e^{i n(\theta-\gamma)}
$$

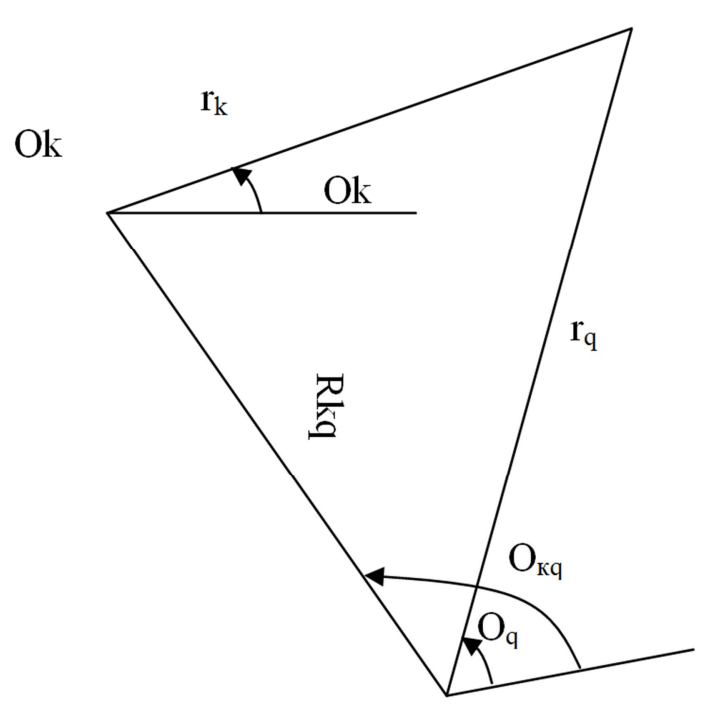

Figure 3. The scheme to the addition theorem.
Unknown coefficients $A_{n}-G_{n}$ are determined from the boundary conditions. As a result, an infinite system of linear equations is obtained, which is solved by an approximate reduction method, provided that the relation is not satisfied.

$$
k \delta(1 \mp \cos \gamma)=2 \pi n
$$

The general characteristic of the program is designed for multi-threaded pipes in the embankment for the case of a drop in seismic waves perpendicular to the axis passing through the tube centers.

The information entered contains the minimum necessary data: the elastic characteristics ( $E$ and $v$ ) of the soil of the embankment and the pipes; density of soil, pipes and liquids filling it; internal and external pipe radii; the predominant period of oscillation of soil particles; coordinates of the point where the VAT is sought; coefficient of seismicity. With the help of a special label, it is possible to count both pipes filled with an ideal compressible fluid and empty ones. The calculation of the cylindrical Bessel and Henkel functions is carried out according to known formulas. The solution of the system of linear equations is carried out by the Gauss method with the separation of the principal term.

Effect of distance between pipes. In Table. 3 the values of the coefficient $\eta_{\max }\left(\eta_{\max }=\left|\sigma_{r r}\right| /(\lambda+2 \mu) \alpha^{2} A\right.$ the maximum radial pressure of the soil on the pipes at a different distance in the light $\mathrm{d}$ between them in the case of a P-wave fall. In this case, the wave number of the $P$ wave $\alpha_{r}=1,0$ : inner and outer radius of pipes $R_{0}=0,8 \mathrm{~m}$ and $\mathrm{R}=1,0 \mathrm{~m}$ : the predominant period of oscillation of soil particles is $\mathrm{T}=0.2 \mathrm{sec}$. Soil Characteristics: Permanent Lamé $\lambda_{1}=8,9-\mathrm{MPa} ; \mu_{1}=4,34 \mathrm{MPa}$; density $\rho_{1}=1,74 \mathrm{Kn} \mathrm{sec} / \mathrm{m}^{4}$. Pipe material characteristics $\lambda_{2}=8690 \mathrm{MPa} ; \mu_{2}=12930 \mathrm{MPa} ; \rho_{2}=2,55 \mathrm{Kn} \mathrm{sec}{ }^{2} / \mathrm{m}^{4}$.

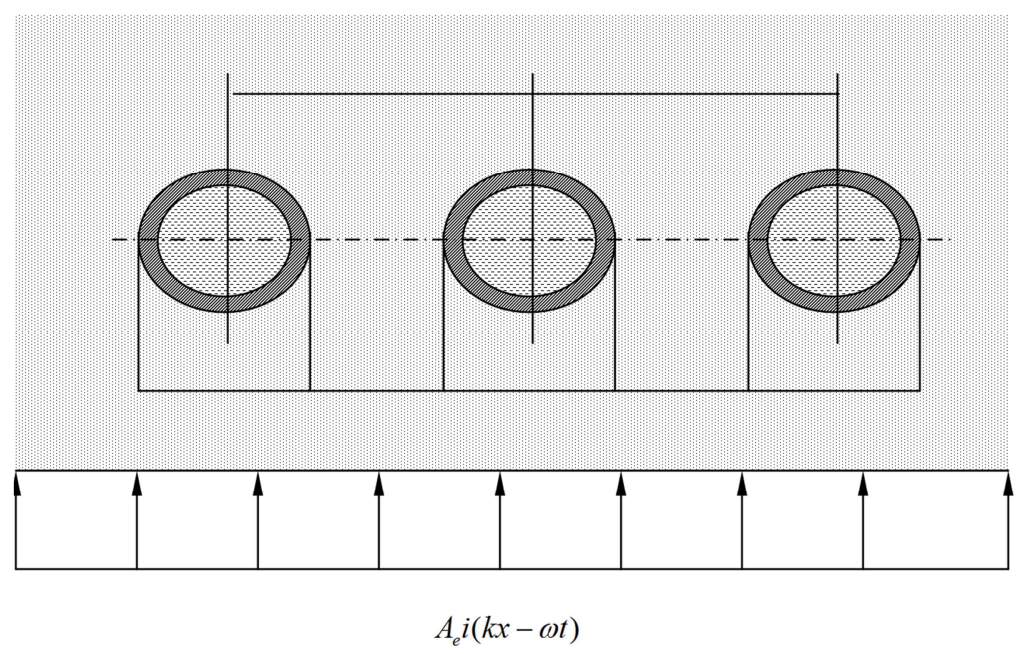

Figure 4. The calculation scheme.

Table 3. The value of the coefficient of dynamic concentration at different distances between the tubes for the case of P-wave incidence.

\begin{tabular}{lllll}
\hline $\mathbf{D} / \mathbf{d}$ & $\mathbf{0 , 5}$ & $\mathbf{1 , 0}$ & $\mathbf{2 , 0}$ & $\mathbf{4 , 0}$ \\
\hline$\eta_{\max }$ & 1,68 & 1,76 & 1,61 & 1,60 \\
\hline
\end{tabular}

From Table 3 it follows that first with increasing the distance between the pipes $0,5 \leq \mathrm{d} / \mathrm{D} \leq 1,0$ coefficient $\eta_{\max }$ slightly increases by $5 \%$, and with further increase $d / D>$ 1.0 decreases more sharply by $10 \%$. When $d / D>2,0$ value $\eta_{\max }$ stabilizes, i.e. practically does not change, with $1 \leq 4,0$ close to the value $\eta_{\max }$ for a single pipe according to calculations. 
Consequently, the mutual influence of reinforced concrete pipes of multiline stacking takes place with the distance between them $\mathrm{d} \leq 4,0 \mathrm{D}$ and leads to an increase in the maximum dynamic pressure of the soil on them compared to a single pipe. This effect of increasing the coefficient $\eta_{\max }$ is associated with the imposition of waves reflected by several surfaces of multicell pipes. In this case, the no monotonic increase in the coefficient $\eta_{\max }$ with a decrease in the distance between the tubes, $d / D$ is connected in our opinion with the phenomenon of interference of superimposed waves after reflection.

This phenomenon is extremely important for the practice of designing seismic underground multiline pipelines, since allows you to choose the optimal distance between the pipes, in which the dynamic pressure during seismic action is minimal. For example, in Table 3 , such a distance is $d=0.5 \mathrm{D}$. It is well known for comparison that in the case of static action, the reverse is observed: the ground pressure on the multicell pipes is less than the single pressure.

In addition to the foregoing, when analyzing the effect of the distance between pipes on their VAT, it is necessary to take into account the relation (4.28), (the so-called "slip points"), at which a significant increase in dynamic stresses in the vicinity of the tube-resonance is observed. This phenomenon is known from optics called Wood's anomaly is a feature of the multi-threaded pipeline and can not arise in a pipeline laid in one thread. From the point of view of design practice, it is necessary to know at what distance it is possible to lay pipes so that a dangerous phenomenon does not occur to resonance.

The answer to this question is given by the relation (5). Let us analyze this relation for the case of the action of $\mathrm{P}$ and $\mathrm{SV}$ seismic waves on a subterranean pipeline. Table 4 shows the dependence of the maximum distance in the light between the centers of the tubes $d_{\max }$, at which there is no resonance, from the angle of incidence of seismic waves $\gamma$.

Table 4. Dependence of distance $D_{\max }$ from the angle of incidence $\gamma$.

\begin{tabular}{llllllll}
\hline $\boldsymbol{\gamma}$. Grad & $\mathbf{0}$ & $\mathbf{3 0}$ & $\mathbf{4 5}$ & $\mathbf{6 0}$ & $\mathbf{7 0}$ & $\mathbf{8 0}$ & $\mathbf{9 0}$ \\
\hline $\mathrm{D}_{\max }, \mathrm{M}$ & 5,0 & 5,36 & 5,86 & 6,66 & 7,45 & 8,52 & 10,0 \\
\hline
\end{tabular}

It follows from Table 4 that the smaller the angle of incidence of a seismic wave on a pipeline, the closer one must lay pipes to each other. Thus, the appearance of resonance in multi-threaded pipes can be avoided by choosing the appropriate distance between them and, thereby, ensuring the seismic stability of the pipeline. Influence of the type of seismic action (P-, SV- or SH-wave). Table 5 lists the values $\eta_{\max }$ of the maximum radial pressure of the soil on the pipes in the event of a fall in the P-and SV-seismic waves at different distances $d$ in the light between the pipes.

Table 5. Coefficient value $\eta_{\max }$ with seismic actions in the form of $P$ and $S V$ waves at different distances $d$ between the pipes.

\begin{tabular}{lll}
\hline $\mathbf{d} / \mathbf{D}$ & $\boldsymbol{\eta}_{\max }$ & \\
\hline & $\mathbf{P}$ - wave & SV - wave \\
1,0 & 1,76 & 1,29 \\
2,0 & 1,61 & 1,72 \\
4,0 & 1,60 & 1,51 \\
\hline
\end{tabular}

At the same time, $\beta_{\mathrm{r}}=2$. Analysis of the data of Table. 5 shows that for $\mathrm{d} / \mathrm{D}<4.0$ the coefficient values $\eta_{\max }$ For the $\mathrm{P}$-wave and $\mathrm{SV}$-wave is as if in antiphase, i.e. at $1 / \mathrm{D}=1.0$, the maximum seismic effect of the $\mathrm{P}$-wave is $27 \%$ higher than for the SV wave, at $\mathrm{d} / \mathrm{D}=2.07 \%$ lower, and at $\mathrm{d} / \mathrm{D}=$ 4.0 again higher, but only by $1 \%$.

At the same time, as the distance between the pipes increases, the difference in these effects decreases and at $\mathrm{d} /$ $\mathrm{D}=4.0$ it practically disappears altogether. In addition, we note that when an $\mathrm{SV}$-wave is applied, the values $\eta_{\max }$ at different distances between the pipes has a 2.5 times greater spread (up to $25 \%$ ) than when the $\mathrm{P}$ wave is applied (up to $10 \%$ ). Thus, the phenomenon of "local resonance" manifests itself more strongly for seismic action in the form of an SV wave.

Influence of fluid filling pipes. Table 6 shows the values of the coefficient $\eta_{\max }$ in the case of a fall of P-wave on empty and water-filled pipes at different distances $d$ in the light between the pipes. The density of the liquid was assumed equal to $\rho_{3}=0,102 \mathrm{Kn} \mathrm{sec} 2 / \mathrm{m}^{4}$.

Table 6. Coefficient value $\eta_{\max }$ for the case of the fall of P-wave on empty and water-filled pipes.

\begin{tabular}{lll}
\hline $\mathbf{d} / \mathbf{D}$ & $\boldsymbol{\eta}_{\max }$ & \\
\hline & P - wave & SV - wave \\
1,0 & 1,76 & 1,89 \\
2,0 & 1,61 & 1,78 \\
4,0 & 1,60 & 1,90 \\
\hline
\end{tabular}

From Table 6 it follows that the presence of water in the pipes increases the seismic effects on them compared to empty pipes. Obviously this is due to the increase in the mass of the pipeline. The maximum dynamic pressure of the soil on the pipes is enhanced. For example: for $\mathrm{d} / \mathrm{D}=1.0$, the difference in the values of the coefficient $\mathrm{d} / \mathrm{D}=2.0-10 \%$, with $\mathrm{d} / \mathrm{D}=4.0-19 \%$.

In addition, we note that the spread in the values of the coefficient $\eta_{\max }$ at different distances $d$ for pipes filled with water less $(7 \%)$ than for empty pipes $(10 \%)$.

Influence of the length of the incident seismic wave. Table 6 shows the coefficient values $\eta_{\max }$ different lengths $l_{0} / l_{0^{-}}$ $2 \pi / \alpha, p$ - wave incident on empty pipes, located at a distance $1=1,0 \mathrm{D}$ from each other.

Table 7. Values of the coefficient $\eta_{\max }$ for different lengths $l_{0} P$ - waves.

\begin{tabular}{llll}
\hline $\mathbf{l}_{\mathbf{0}} / \mathbf{D}$ & $\mathbf{3 , 0}$ & $\mathbf{5 , 0}$ & $\mathbf{1 0 , 0}$ \\
\hline$\eta_{\max }$ & 1,76 & 1,52 & 1,20 \\
\hline
\end{tabular}

From Table. 7 it follows that the greater the length of the incident seismic wave, i.e. The denser the soil of the embankment, the lower the coefficient $\eta_{\max }$. For reference, we note that relation $l_{0} / D=5,0$ - not in bulk sand, sandy loam and loamy soil; $l_{0} / D=10,0$ - clay soils.

Thus, the type of soil, and especially its density, has a significant effect on its dynamic pressure on the pipes under seismic action.

Hence it follows that when erecting a mound over pipes, it is necessary to carefully compact the bulk ground. It is 
interesting to note that a good compaction of the soil can also reduce its static pressure on the pipes. In addition, the calculations show that when $1_{0}>10,0 \mathrm{D}$ The dynamic problem reduces to a quasistatic problem, which essentially simplifies its solution. From this follows the important conclusion 0 that the quasistatic approach is not applicable to the calculation of the seismic effect of pipes under embankments.

Effect of wall thickness of pipe and concrete class. Table 8 shows the values of the coefficient $\eta_{\max }$ for different thicknesses of the wall of the reinforced concrete pipe in the event of a fall of the P-wave into empty multi-threaded pipes, stacked multi-threaded pipes laid at a distance $d=0,5$.

Table 8. Coefficient value $\eta_{\text {mах }}$ для different pipe wall thicknesses $t$.

\begin{tabular}{lllll}
\hline $\mathbf{d} / \mathbf{D}$ & $\mathbf{0 , 0 8}$ & $\mathbf{0 , 1}$ & $\mathbf{0 , 1 5}$ & $\mathbf{0 , 2}$ \\
\hline$\eta_{\max }$ & 1,60 & 1,66 & 1,66 & 1,68 \\
\hline
\end{tabular}

From Table. 8 it follows that the range of wall thickness, which are produced by domestic industry reinforced concrete pipes, practically does not affect the dynamic pressure of the soil, not these pipes. This, in all likelihood, is due to the fact that the seismic wave does not penetrate the reinforced concrete pipe due to the sufficient rigidity of the pipe.

A similar conclusion, having the same reasons, can be obtained from Table 9, in which the values $\eta_{\max }$ for various classes of concrete used for the manufacture of pipes, with a wall thickness $\mathrm{t}=0,1 \mathrm{D}$.

Table 9. Coefficient value $\eta_{\max }$ for various classes of concrete.

\begin{tabular}{lllll}
\hline Class of concrete & B20 & B30 & B40 & B50 \\
\hline$\eta_{\max }$ & 1,68 & 1,68 & 1,67 & 1,68 \\
\hline
\end{tabular}

1. With seismic action, the mutual influence of reinforced concrete pipes of multiline stacking takes place with a distance in the light between them $\mathrm{d}>4,0 \mathrm{D}$ and leads to an increase in the maximum dynamic pressure of the ground on them as compared to a single pipe (local resonance phenomenon) by $5-10 \%$.

2. The appearance of resonance in multicell pipes can be avoided by choosing the distance between them to the nonmultiple length of the incident seismic wave. This phenomenon of resonance is a feature of the multi-threaded pipeline and can not occur in a pipeline laid in a single string.

3. The phenomenon of local resonance manifests itself more strongly for seismic action in the form of SV-wave than P-waves.

4. The presence of water in the pipes increases the seismic effect on them by $10-20 \%$.

5. The thicker the soil of the embankment, the less seismic impact on underground pipes. For $\mathrm{l}>10 \mathrm{D}$, the dynamic problem reduces to a quasistatic problem.

6. The change in wall thickness and class of concrete practically does not affect the dynamic pressure of the soil on reinforced concrete pipes under seismic action.

\section{Conclusion}

The materials considered allow us to draw the following main conclusions.

1. Accidents of underground structures during earthquakes indicate the need for more careful consideration of seismic loads during design. Reliable instrumental records of earthquakes at considerable depths are practically absent at present. The question of the intensity and spectral composition, the nature of the attenuation of seismic influences at various depths and in specific ground conditions remains unclear. In this regard, the acquisition of seismological information for underground conditions is necessary to address the issues of underground seismic resistant construction.

2. The methods of recording seismic loads on underground structures developed and currently used in accordance with the static seismic stability theory do not take into account a number of important factors and can give underestimated stresses. In the calculation of working systems, when interference phenomena, the application of static theory can lead to significant errors.

3. The question of the influence of the free surface and the relief of the terrain on the seismic stress of underground structures has not been studied at all. In this case, too, the contribution of waves reflected from the free surface.

4. Spectral methods of the theory of seismic resistance have not found wide application in the calculation of underground hydraulic structures in the absence of valid accelerometer spectra, as well as the lack of techniques for determining the frequencies and patterns of natural oscillations of such structures.

5. Methods of wave dynamics have made it possible to solve a number of plane problems on stress concentrations in a linearly elastic formulation. The obtained results show that when seismic waves are applied to structures, a complex field arises that cannot in principle be studied by other methods. Not taking into account the visco elastic properties of soils in the calculation of underground structures for seismic impacts can in some cases lead to significant errors.

6. The methods currently used to ensure the safety of underground structures during seismic explosions are based on empirical formulas obtained as a result of field observations, they need correction and justification. When solving the problems of the strength of structures in earthquake conditions, it is necessary to use deformation-strength characteristics of materials, determined under conditions corresponding to the frequencies of natural effects. In this regard, standard techniques should be developed.

7. Under the influence of harmonic waves on a cylindrical body in an elastic medium, the values of displacement and stress are obtained by solving an algebraic equation with complex coefficients in an analytical form. The problems of the proper flat and antiplane vibrations of an infinitely elastic cylinder with external friction at the boundary are formulated and 
investigated. The forced antiplane vibrations of the elastic semi-infinite cylinder with external friction at the boundary are formulated and investigated. It is established that for all the considered parameters of external friction, including for $\alpha=1$, the error is of the order of one to two percent.

8. It is established that for the first mode of motion the effect of the medium on the oscillation frequency is particularly important for relatively thin shells $(\chi<0.03)$, for shell thickness $\chi<0,07$, the obtained values of the frequency practically coincide with the exact values.

9. A method has been developed for determining the coefficient of friction and the frequency of natural oscillations of cylindrical bodies for the application of specific parameters, $E, v, \rho$. It is established that the coefficient of friction decreases with increasing diameter of the tube. A reduction in soil stiffness reduces stress in the pipeline.

Based on the study of the diffraction of harmonic elastic waves with respect to a reinforced cavity in a continuous elastic medium, it is established that in the region of low and medium frequencies $1 / 2 \leq k_{1} \alpha \leq 2 \div 3$, the medium exerts the maximum both inertial and damping effects on the motion of the cylindrical system. When the pipeline is insulated with soft ground, it is established that the insulation is effective only for high frequencies.

\section{References}

[1] Avliyakulov NN, Safarov II Modern problems of statics and dynamics of underground pipelines. Tashkent, publishing house: Fan va texnologiya. 2007. 306 pp.

[2] Avliyakulov NN, Safarov II. Methods of increasing the seismic resistance of underground plastic pipelines // Journal of Oil and Gas, 2005, no.44S.42-44.3. Aptikeyev F. F. Seismic oscillations in earthquakes and explosions.-M.: Science, 1969.-104 p.

[3] Aleshin V. V. [and others] Numerical analysis of the strength of underground pipelines. Moscow: URSS Publishing House, 2003. 320 p.

[4] Bazhenov VA Bending of cylindrical shells in an elastic medium.-Kiev. Publisher: Graduate School, 1975.-186p.

[5] Baron, Matthews. Diffraction of a pressure wave with respect to a cylindrical cavity in an elastic medium. // Applied mechanics. No. 11, 1961. p.229-237.

[6] Bozorov M. B., Safarov I. I., Shokin Yu. I. Numerical simulation of oscillations of dissipatively homogeneous and inhomogeneous mechanical systems.-Novosibirsk, 1996.-187 p.

[7] Bozorov M. B., Safarov I. I., Troyanovsky I. E. On the natural oscillations of inhomogeneous mechanical systems. // Uzb. Journal of Problems of Mechanics, No. 3-4. 1994. p.3-6.

[8] Bykhovsky VA, Zavriev S. and others, Seismic resistant constructions abroad.-M.: Stroiizdat, 1968.-221 p.

[9] Watson G. N. Theory of Bessel functions, Ch. 1-M., 1949.$798 \mathrm{p}$.
[10] Volmir A. S. Shells in the flow of liquid and gas. // Aero elasticity problem.-Moscow: Science. 1976.-416 p. Ger.

[11] Galiev Sh. U. Dynamics of the interaction of structural elements with a pressure wave in a liquid.-Kiev: ScienceDumka. 1977.-172 p.

[12] Goldenblat I. I., Kartsivadze G. N. Napetvaridze Sh. G., Nikolaenko N. A. Designing of seismic resistant hydraulic, transport and other structures.- M.: Stroiizdat, 1971.280 p.

[13] Gorshkov A. G. Dynamic interaction of shells and plates with the environment.-Moscow: Izd. AN. Mechanics of a solid., No. 2, 1976. p.165-178.

[14] Grinchenko V. T., Meleshko V. V. Harmonic oscillations and waves in elastic bodies.-Kiev: Science-Dumka, 1981.-284 pp.

[15] Gontkevich V. S. Own oscillations of spherical shells. In the book. Studies on the theory of structures. Issue 13,-Moscow: Gosstroyizdat. 1964. p.77-83. ger.

[16] Guz A. N., Golovchan V. T. Diffraction of elastic waves in multiply connected bodies.-Kiev: Science-Dumka, 1972.-254p.

[17] Guz A. N. Propagation of waves in a cylindrical shell with a viscous compressible fluid. // Applied Mechanics., No. 10, 1980. p.10-20.

[18] Dashevsky M. A. Diffraction of elastic waves on a plane supported by a ring of rigidity. Construction mechanics and calculation of structures. No. 2, 1967. p. 33-36.

[19] Erzhanov N. S., Aitaliev J. M., Masanov Zh. K. Seismic stress of underground structures in an anisotropic massif.-Alma-Ata: Science. 1980.-211p.

[20] Zavriev K. S., Nazarov A. G., Eisenberg Ya. M. et al. Fundamentals of the theory of seismic resistance of buildings and structures.-Moscow: Stroiizdat, 1970.-224 p.

[21] Ilgamov M. A., Ivanov V. A., Gulin B. V. Strength, stability and dynamics of shells with elastic filler.-M. 1977.-332p.

[22] Isroilov M. Sh. Dynamic theory of elasticity and wave diffraction.-Moscow: Izd. MSU.1992.-206 p.

[23] Kabulov V. K. Algorithmization in the theory of elasticity.Tashkent: Fan. 1968.-394 p.

[24] Koltunov M. A., Mirsaidov M., Troyanovsky I. E. Steady oscillations of axisymmetric viscoelastic shells. // Mechanics of polymers. No. 4, 1978. p.290-315.

[25] Kruse-pascal D., Gernet T., Pifko D. Effect of viscoelasticity of the environment on unsteady reaction of round cylinders of arbitrary thickness under the action of plane waves. // Applied mechanics; translation from English, v.34, ser. E, No. 2, 1967. p.120-128.

[26] Kubenko V. D., Kuzma V. M., Puchka G. N. Dynamics of spherical bodies in a fluid under vibration.-Kiev, 1987.-154 p.

[27] V. D. Kubenko. Nonstationary interaction of structural elements with the environment.-Kiev: Science-Dumka, 1979.$183 \mathrm{p}$.

[28] Mau Mente. Dynamic stresses and displacements in the vicinity of the cylindrical surface of discontinuity from a plane harmonic shear wave. // Applied mechanics, translation from English, vol.30, ser E, No. 3, 1963. p.117-126. 
[29] Mironov P. S. Explosions and seismic safety of structures.M.: The science, 1973.-168 p.

[30] Mostkov V. M. Underground structures of large cross section.M.: The science, $1974 .-320 \mathrm{p}$.

[31] Mohnachev M. P., Gromova N. V. Fatigue of rocks at a stretching.- Physical and technical problems of working out of minerals, 1975, №3, p.49-53.

[32] Muborakov Ya. N. Seismic dynamics of underground structures such as shells.-Tashkent: Fan. 1987.-192 p.

[33] Напетваридзе Sh. G. Seismic stability of hydraulic structures.-Moscow: Stroyizdat, 1959.- 216 p.

[34] Okomoto Sh. Seismic stability of engineering structures.Moscow: Stroyizdat. 1980.-342p.

[35] Ostroverkh B. N. Problems of calculating tunnels under seismic action. // In the book. Dynamics of foundations, foundations and underground structures. t. 2.-Tashkent, 1977. p. $98-103$.

[36] Otpuschennikov E. N., Lovkov S. Ya., Kostin I. Kh. An experimental study of the stress concentration near a circular hole under the influence of a plane compression wave. Stress concentration. Kiev, 1971, vol. 3, p.106-112.

[37] Penkovsky V. G. Interaction of low-rigidity lining with surrounding soil in underground structures with dynamic effects from earthquakes.-In the book. Waves in soils and vibrometry issues. Proceedings of the Third All-Union Conference on Dynamics of Foundations, Foundations and Underground Structures. Tashkent, 1975, p. 67-71.

[38] Rashidov T. R. Dynamic theory of seismic resistance of complex systems of underground structures.-Tashkent: Fan. 1973. $-182 \mathrm{p}$.

[39] Rashidov T. R., Dorman I. Ya., Ishankhodjaev A. A. Seismic stability of tunnel structures of subways-M.: Transport. 1975.$120 \mathrm{p}$.
[40] Rashidov T. R., Khomzhatov G. Kh., Mardonov B. M. Vibrations of structures interacting with the ground.-Tashkent: Fan. 1975.-174 p.

[41] Savin G. N. Stress distribution near the holes.-Kiev: ScienceDumka. 1968.-887 p.

[42] Safarov II, Teshaev M. Kh., Kilichev O. Dynamic stressed states of thin-walled pipelines.. LAP LAMBERT Academic Publishing Saarbrucren Dentschland / Germanu /-2015-230p.

[43] Safarov I. I., Boltaev Z. I., Akhmedov M. Distribution of the natural waves. LAP LAMBERT Academic Publishing Saarbrucren Dentschland /Germanu/-2015.-110p.

[44] Safarov I. I. Vibrations and waves in dissipative inhomogeneous media and structures.-Tashkent: The science. 1992.-250 p..

[45] Urazboev M. T. Seismic stability of elastic and hydroelastic systems.-Tashkent: The science, 1968.- $254 \mathrm{p}$.

[46] Filippov IG, Egorov OA Nonstationary oscillations and diffraction of waves in acoustic and elastic media. // M.: Mechanical Engineering, 1977.- 303p.

[47] Shulman S. G. Calculations of seismic resistance of hydraulic structures with allowance for the influence of the wave medium. /-M: Energy, 1976. -- 336 p.

[48] Pao Y. H., Mow C. C. diffraction of elastic waves and dynamic stress concentration. Grane, Russak, 1973. 694 p.

[49] Yoshizaki K., Rouke T. O., Hamada M. Large scale experiments of buried steel pipelines with elbows subjected to permanent ground deformation // Structural Eng. Earthquake Eng., JSCE. 2003. Vol. 20. Pp. 1-11.

[50] Zdanchuk E., Lalin V. The theory of continuum medium with free rotation without coupled stresses // Proc. of the XXXVIII Summer School-Conference advanced problems in mechanics. SPb, 2010. Pp. 771-775. 